darstellungen erklären zu können. Dabei setzt sie mit dem gesellschaftlichen Kontext als diskursive Aktivität der Rezipierenden und den Geschlechterpositionen zwei Schwerpunkte.

Es bleibt zu fragen, inwieweit ihre vorab entwickelten Thesen ihre Auswertung von Gruppendiskussionen beeinflussten. Die moderierten Diskussionen mit Frauen, Männern und gemischten Gruppen über zwei ausgewählte Gewaltszenen des fiktionalen Fernsehprogramms zeigen geschlechterspezifische Rollenidentifikationen mit Täter oder Opfer, Reaktionen auf Gewaltdarstellung ebenso wie implizite Vorstellungen über Geschlechterrollen und adäquates Geschlechterverhalten. Die Szenenauswahl setzt bewusste Differenzen: Es handelt sich um eine hegemoniale Szene, „in der die Gewalt dem gesellschaftlichen Machtverhältnis folgt" und eine nicht-hegemoniale Szene, „in der ebenfalls eine Frau bedroht wird, diese ihren Angreifer jedoch besiegt." (13) Bei der Auswertung werden einige besonders aussagekräftige Zitate aus den Gruppendiskussion in ganz unterschiedlichen Analysezusammenhängen mehrfach verwertet.

Röser konstatiert als Untersuchungsergebnis einen „tiefgreifenden Einfluss der Kategorie Geschlecht im Gewaltzusammenhang." (350) Das Geschlechter-Gewalt-Verhältnis sei in „so eindeutiger Weise strukturiert, wie man es in Zeiten gesellschaftlicher Uneindeutigkeit nur selten findet." Dabei sind besonders jene Dominanzverhältnisse einflussreich, „die Ohnmachtpositionen mit Körperlichkeit verbinden und begründen." (350)

Die Studie ist ein exemplarisches Beispiel für die Notwendigkeit interdisziplinärer Medienforschung. Durch sie ließe sich die von Werner Früh konstatierte "wechselseitige, dynamische Abhängigkeit von Medienbotschaft und Publikumswahrnehmung angemessen“ (Früh 1995, 173) berücksichtigen. Die Integration medienund filmwissenschaftlicher Studien $\mathrm{zu}$ den ökonomischen Rahmenbedingungen der Produktion, zur Genreforschung und zur Geschlechterdarstellung hätten noch weitere Erklärungsmuster liefern können. Auf diese Weise ließe sich Rösers Auffassung von Medientexten als „symbolische Repräsentationen gesellschaftlicher, sozialer, kultureller Themen" (343) stärker untermauern. Die Autorin selbst spricht „vom Zusammenspiel von Eigenschaften des Produkts und Konstruktionsleistungen der Rezipierenden." (37) und konsta- tiert: „Erst wenn der Aneignungsprozess im Spannungsfeld von Text und Kontext in die Analyse integriert wird, kann die Frage nach Wirkungen sinnvoll gestellt werden." (38)

So ist die Gewaltdarstellung gegen Frauen genrespezifisch unterschiedlich. Viele in unterschiedlichen Zusammenhängen ausgewertete Beiträge der Probanden, etwa ihre Prognosen über den Handlungsverlauf der Filme aus denen die Beispielsszenen stammen, lassen sich auf ihre durch lange Seherfahrung erworbene Genrekenntnis zurückführen. Zwar konstatiert Röser eine erstaunliche Medienkompetenz ihrer Gesprächsteilnehmer, geht aber selbst nur rudimentär auf Genrekonventionen ein. So bezieht sich die häufig thematisierte Komik des Rollenwechsels in einer Gewaltszene vor allem auch auf Genresspezifika des Gendercrossing etwa in Komödien. Siegfried Kaltenecker hat in „Spiegelformen. Männlichkeit und Differenz im Kino“ (Basel, Frankfurt am Main 1996) die Genrekonventionen dieses Rollenwechsels untersucht. Für die Gesprächsanalyse wären ergänzende Untersuchungen aus dem Bereich Cultural Studies, etwa Andreas Hepps Studie zur Fernsehaneignung in Alltagsgesprächen, hilfreich gewesen.

Röser selbst versteht ihren eigenen Ansatz zur Analyse von Fernsehgewalt und ihrer Wirkung als Verabschiedung „von der Suche nach dem objektiven Gehalt medialer Gewalt und nach globalen Wirkungen“ (342) Für die künftige Forschung schlägt Röser die stärkere Berücksichtigung von Themen ,wie Macht und Marginalisierung, soziale Konflikte und Wandlungsprozesse als Kontext der Mediengewaltrezeption vor" (348). Mit ihrer Studie hat sie einen wichtigen Ausgangspunkt für neue Perspektiven in der Gewaltforschung geleistet.

Joan Kristin Bleicher

\section{Christoph Bieber}

\section{Politische Projekte im Internet}

Online-Kommunikation und politische Öffentlichkeit

Frankfurt/New York: Campus, 1999 - 232 S.

ISBN 3-593-36344-5

Das Phänomen „Internet“ stellt mehr noch als für Bürger und Politiker eine Herausforderung sowohl für die Politik- als auch für die Kommunikationswissenschaft dar: Das Problem 
beginnt bereits bei der Frage, ob das Internet etwa ein "Medium“ im kommunikationswissenschaftlichen Sinne sei, und wodurch sich Online-Kommunikation von herkömmlicher interpersonaler und Medienkommunikation unterscheide. Christoph Bieber hat mit seinem Buch die Aufgabe übernommen, der OnlineKommunikation zumindest im begrenzten Ausschnitt der politischen Kommunikation eine wissenschaftliche Struktur zu geben. Ziel der Arbeit ist es, mit einem explorativ angelegten Vorgehen „eine detaillierte Analyse der im Entstehen begriffenen Orte sichtbaren politischen Handelns im Netz" zu liefern, sowie auf der Basis einiger Fallstudien auch die „theoretische Fragestellung nach einer Transformation der politischen Öffentlichkeit“ (20) im Internet zu erörtern.

In Kapitel 2 umreißt Bieber den für seine Arbeit zentralen Begriff der Online-Kommunikation aus kommunikationswissenschaftlicher Sicht. Dabei wird gleich zu Beginn klargestellt, dass es die spezifischen Eigenschaften von Online-Kommunikation nicht geben kann. Vielmehr weisen verschiedene Online-Kommunikationsformen (E-Mail, Chats, etc.) ganz unterschiedliche Merkmale auf, die jeweils im Einzelfall für deren wissenschaftliche Definition herangezogen werden müssen. Bieber versucht sinnvollerweise aber ohnehin nicht, eine endgültige und umfassende Definition von Online-Kommunikation zu liefern; ihm kommt es vielmehr darauf an, aus den bereits existierenden Fragmenten theoretischer Begriffsdefinitionen (Morris/Ogan 1996; Rössler 1998) ein Instrumentarium zur intramedialen Unterscheidung verschiedener Online-Angebote zu erarbeiten. Für ihn ist dabei das Merkmal der „Interaktivität“ zentral, das er in vier Dimensionen ausdifferenziert, die ihm im Weiteren als Folie für die Analyse verschiedener politischer Online-Projekte dienen.

Die darauf folgende Beschreibung der „Mechanismen und Strukturen" der Online-Kommunikation ist, obwohl der Text immerhin erst 1999 veröffentlicht wurde, weitgehend überholt. Das ist allerdings nicht dem Autor, sondern vor allem der rasanten Entwicklung sowohl in der Technik als auch der sozialen Aneignung des Internets anzulasten. Ebenso wie das Glossar am Ende des Buches dienen die in diesem Abschnitt vorgestellten Definitionen der wichtigsten Elemente der Netzkommunikation wie „Links“, „Suchmaschinen“ oder die
Darstellung der „aktuellen“ Verbreitung der Internetnutzung eher als Einstiegslektüre für wissenschaftliche Internet-Neulinge. Davon dürfte es in der Zielgruppe des Buches heute jedoch nur noch wenige geben.

Theoretisch eingegrenzt wird Biebers Analyse der Online-Kommunikation in Kapitel 3 durch das Konzept der "Öffentlichkeit“, das eine zentrale Rolle in der Beziehung von Medien und Politik spielt. Nahe gelegt wird dies durch die oft gebrauchte Metapher des Internets als „Cyberspace“, dem die Öffentlichkeit als nach Habermas'scher Definition „im kommunikativen Handeln erzeugter sozialer Raum" gegenüber steht. Inwieweit im virtuellen Raum des Internets also Öffentlichkeit entsteht - und ob sie dabei in Form einer spezifischen „Netzöffentlichkeit" auftritt - ist die Frage, die durch die Analyse dreier „politischer Online-Projekte“ im nächsten Abschnitt geklärt werden soll. Der Hebel, an dem Bieber ansetzt, sind die Akteure der Öffentlichkeit, die nach Peters (1993) in Akteure des Zentrums, der inneren und der äußeren Peripherie differenziert werden.

Im Kapitel 4, dem umfangreichsten des Buches, folgen die Analysen von drei „politischen Projekten“ im Internet. Methodisch handelt es sich dabei um eine inhaltsanalytische Untersuchung von im WWW veröffentlichten Angeboten verschiedener politischer Akteure. Obwohl in Kapitel 3 das Spektrum möglicher politischer Akteure und ihrer Online-Aktivitäten aufgefächert wurde, dient diese Strukturierung nicht zur Auswahl der konkreten Fallbeispiele. Als Kriterien erscheinen hier die drei Begriffe „Entstehung“, „Herstellung“ und „Besetzung von Öffentlichkeit im Netz" auf. Damit kommen weitere Dimensionen ins Spiel, deren Einordnung in das Konzept der Untersuchung der Leser vermisst. Andererseits verwendet der Autor viel Sorgfalt auf eine detaillierte Untersuchung seiner drei Fallbeispiele: virtueller Parteizentralen, Online-Wahlkämpfe und politischer Proteste im Internet (Blue Ribbon Campaign, Kryptographie-Kampagne, Hochschulstreik im Internet). Diese Analysen bieten außerordentlich erhellende Einblicke, weil der Autor einerseits stark systematisch vorgeht - etwa indem die Angebote mit einem vorher definierten Kategoriensystem auf Inhalt und Struktur geprüft werden -, andererseits weil die Offenheit dieser explorativen Studie ihm auch die Freiheit 
gibt, auf auffällige Besonderheiten je nach Bedarf einzugehen.

Das abschließende Kapitel 5 dient Bieber zur Synthese der empirischen Erkenntnisse mit dem in Kapitel 3 entwickelten theoretischen Ansatz der „Netzöffentlichkeit“. Deutlich wird dabei eine Entwicklung in zwei gegenläufige Richtungen: Die analysierten politischen Online-Aktivitäten lassen einmal eine stärkere „Verdichtung des Akteursgefüges“ (187) durch eine intensive Vernetzung und interne Kommunikation erkennen, auf der anderen Seite öffnet sich das Netz für neue Akteure und vielfältigere Formen der Kommunikation. Diese Entwicklungen lassen Bieber schließlich vom Internet als "vernetzter Teilöffentlichkeit“ (195) sprechen, die sich durch inter- und intramediäre Vernetzung der Akteure auszeichnet, die in diesem Umfang und in dieser Vielfalt nur durch die vielfältigen Formen der OnlineKommunikation möglich ist. Allerdings zeigt sich auch, dass mit dieser Vielfalt eine Einschränkung insoweit verbunden ist, als die Reichweite der Online-Kommunikation geringer ist als die der herkömmlichen „Massenkommunikation": Es existieren nur noch Teilöffentlichkeiten statt einer allgemeinen Öffentlichkeit.

Christoph Bieber hat mit seinem Buch sicherlich einen Eckstein in der politikwissenschaftlichen Online-Forschung gesetzt, nicht zuletzt deshalb, weil die Literatur in diesem Bereich bisher größtenteils von kleinteiligen Sammelband-Veröffentlichungen dominiert ist und er dem eine nur im Rahmen einer Monographie zu leistende Tiefe in der Analyse politischer Online-Kommunikation entgegengesetzt hat. Besonders wertvoll ist in diesem Zusammenhang sein empirischer Ansatz: Gerade die politikwissenschaftliche Literatur zum Internet erschöpft sich bisher zu häufig in theoretischen Erörterungen des vermeintlichen Potenzials der Online-Kommunikation für Politik und Demokratie. Allerdings kann das Buch nur ein erster Ansatz sein, dem noch viele, evtl. stärker deduktiv angelegte Untersuchungen folgen müssen, um ein klares Bild von der Rolle des Internets für die politische Öffentlichkeit zu gewinnen.

Martin Emmer

\section{Anja Claudia Todtenhaupt}

\section{Cyber TV - Die Digitalisierung der Film- und Fernsehproduktion}

Münster: Lit, 2000. - 322 S.

(Beiträge zur Computersoziologie; 2)

\section{ISBN 3-8258-4921-X}

Virtualität - wenn etwas die Innovationen im Medienbereich prägt, so ist es dieses Phänomen. Es beinhaltet, dass das Wahrgenommene immer mehr immer weniger (wirklich) ist - was dem Beobachter zu erkennen zunehmend schwerer fällt. Der Aspekt der Virtualität sticht bei der (Weiter-)Entwicklung von Medieninhalten nicht besonders hervor, eher im Gegenteil: Auswirkungen der Rezeption dieser neuen Form von „Realitäten“ treten eher unterschwellig auf, wodurch die Gefahr entsteht, dass sie zu wenig reflektiert werden. Ein Grund ist darin zu finden, dass sich die Darstellung virtueller Realität kaum noch von der Wiedergabe von „Wirklichkeit“ unterscheidet. Der zweite Band aus der Reihe der Beiträge zur Computersoziologie, herausgegeben von Dr. Achim Bühl von der Universität Heidelberg, setzt an diesem Punkt an, er will die tief greifenden Veränderungen untersuchen, mitsamt ihren Auswirkungen auf unsere Medienkultur.

Der Buchtitel „Cyber TV“ mag auf wissenschaftlich interessierte Leser leicht abschreckend wirken, handelt es sich doch um einen etwas sehr strapazierten Trend-Begriff. Welches moderne Medienunternehmen schmückt die eigenen Streaming-Aktivitäten im Onlinebereich nicht auch gerne mit diesem schwammigen Begriff, der für Kreativität, Zukunft und Innovation steht/stehen soll? Die Ergänzung des Titels „Die Digitalisierung der Film- und Fernsehproduktion" engt die Blickrichtung des Werkes nur bedingt ein. Man muss wissen, dass sich die Reihe, in der das Buch erschienen ist, mit Computersoziologie beschäftigt, damit klar wird, dass in diesem Band nicht nur die umfangreichen Veränderungen im Film- und Fernsehbereich der letzten Jahre beschrieben werden, sondern sich die Autorin auch kritisch damit auseinander setzt.

Erstaunlicherweise bleibt Anja Claudia Todtenhaupt zu Beginn bei der Definition dieses Begriffs etwas vage, CyberTV wird als (Freizeit-)Phänomen beschrieben (S. 3), das „als ein Element des neuen Medienzeitalters zu gravierenden Veränderungen in unserer Medienkul- 